\title{
Dose-response models for selected respiratory infectious agents: Bordetella pertussis, group a Streptococcus, rhinovirus and respiratory syncytial virus
}

\author{
Rachael M Jones ${ }^{*}$ and Yu-Min Su
}

\begin{abstract}
Background: Dose-response assessment is one step in quantitative microbial risk assessment (QMRA). Four infectious microbes capable of causing respiratory diseases important to public health, and for which dose-response functions have not been available are: Bordetella pertussis (whooping cough), group A Streptococcus (pharyngitis), rhinovirus (common cold) and respiratory syncytial virus (common cold). The objective of this study was to fit dose-response functions for these microbes to published experimental data.

Methods: Experimental infectivity data in human subjects and/or animal models were identified from the peer-reviewed literature. The exponential and beta-Poisson dose-response functions were fitted using the method of maximum likelihood, and models compared by Akaike's Information Criterion.

Results: Dose-response functions were identified for each appropriate data set for the four infectious microbes. Statistical and graphical measures of fit are presented.

Conclusions: With the fitted dose-response functions it will be possible to perform QMRA for these microbes. The dose-response functions, however, have a number of limitations associated with the route of exposure, use of animal hosts, and quality of fit. As a result, thoughtfulness must be used in selecting one dose-response function for a QMRA, and the function should be recognized as a significant source of uncertainty. Nonetheless, QMRA offers a transparent, systematic framework within which to understand the mechanisms of disease transmission, and evaluate interventions.
\end{abstract}

Keywords: Risk assessment, Dose-response, Respiratory infections, Pharyngitis, Common cold

\section{Background}

Quantitative microbial risk assessment (QMRA) is a growing and diversifying area of research for public health. QMRA seeks to evaluate the risk of adverse health effects, particularly infection, resulting for human exposures to infectious microbes. Applications to water-borne and food-borne infectious microbes are too numerous to cite, and have enhanced public health decision making through exploration of: scenarios that cannot be directly observed [1], the effectiveness of interventions [2], and causes of infectious disease outbreaks [3]. In the context

\footnotetext{
* Correspondence: rjones25@uic.edu

Division of Environmental and Occupational Health Sciences, School of Public Health University of Illinois, Chicago, USA
}

of microbes capable of causing respiratory infections, quantitative microbial risk assessment research has focused on influenza [4-8] and tuberculosis, often in the context of transportation [5,9-11]. One reason for limited application of QMRA to microbes capable of causing respiratory infections may be the limited availability of doseresponse functions, which are complicated by uncertainty about mechanisms of disease transmission.

Dose-response functions are mathematical expressions that characterize the probability of an adverse health outcome (e.g., infection) subsequent to an exposure. Doseresponse functions are fitted to adverse health outcomes observed among animals or human volunteers exposed to graded doses of infectious microbes. Though the 
conceptual models underlying dose-response functions differ between microbes and chemical agents [12], the nature of data and use of dose-response functions in risk assessment are similar. Dose-response assessment - the identification and/or development of a dose-response function - is one step in the classical risk assessment paradigm of: hazard identification, dose-response assessment, exposure assessment, and risk characterization. This paper focuses on the development of dose-response functions, and discusses issues around the selection and interpretation of dose-response functions in animal models and exposure routes for human disease transmission. Completion of a QMRA requires substantial additional information and analysis.

Absent a dose-response function, it is possible to apply the concept of the quantum of infection to estimate the risk of infection. This approach is commonly used for Mycobacterium tuberculosis, but has been applied for other infectious microbes transmitted through the inhalation of airborne microbes [5,13-15]. The quantum of infection is an unspecified number of microbes that has the effect of causing infection [16]. If the rate of emission is also measured in quanta of infection, such as when the emission rate is estimated from observed disease or tubercule formation [17], then it is possible to estimate risk without an explicit dose-response function. For example, the Wells-Riley equation may be applied. The Wells-Riley equation estimates the probability of infection among susceptible people as an exponential function of the rate at which quanta of infection are emitted into a well-mixed, ventilated room [18]. Given a single infectious source emitting $q$ quanta per hour, the probability of infection in a susceptible person breathing at rate $p \mathrm{~m}^{3}$ per hour is a function of time ( $t$ hours):

$$
\mathrm{P}(\mathrm{t})=1-\exp (-\mathrm{qpt} / \mathrm{Q})
$$

where $\mathrm{Q}$ is the volumetric airflow rate in the room $\left(\mathrm{m}^{3}\right.$ per hour). As shown in Equation 1, the traditional Wells-Riley model has not included physical processes that remove infectious agents from air - e.g., loss of infectivity and gravitational settling, but the equation can be modified to address these factors [19]. The model need not be limited to steady-state conditions [20]. A greater limitation of the Wells-Riley equation is exclusive consideration of inhalation exposures. Respiratory infections, however, depending upon the microbe, may arise via multiple routes, motivating the need for dose-response functions.

The U.S. Centers for Disease Control and Prevention (CDC) defines three routes of disease transmission: airborne, contact, and droplet [21]. Airborne transmission involves infectious microbes in small particles that remain infectious over time, allowing dispersal of infectious microbes through the air over long distances. Contact transmission involves the transfer of infectious microbes from one infected person to another, though the transmission may be indirect, via a contaminated object. Droplet is a form of direct contact transmission involving the transfer of infectious microbes in droplets (e.g., relatively large particles) projected from the respiratory tract to the mucosal surfaces of a susceptible person, generally over short distances. These definitions do not represent the full spectrum of pathways by which diseases are transferred. An obvious gap is that droplet-generating activities also produce small particles that can be inhaled in proximity to the infectious person, not just at a long distance. Brosseau and Jones have suggested that aerosol transmission replace, or at least bridge, the definitions of droplet and airborne transmission [22]. In addition, the route of disease transmission refers to the primary route (s) for an infectious agent, though selected settings - e.g., laboratories or healthcare settings - may create opportunities for infection through atypical routes [23]. Accurate representations of the transmission routes are necessary for QMRA, as they drive the assessment of exposure and selection of dose-response function.

The objective of this study is to identify dose-response functions for selected infectious agents capable of causing respiratory infections: Bordatella pertussis, rhinovirus, respiratory syncytial virus, and group A Streptococcus. To our knowledge, dose-response functions for these microbes have not been published to date, though relevant data exist. These four microbes persist as threats to public health, such that identification of dose-response functions could facilitate exploration of disease transmission and interventions through risk assessment.

\section{Methods}

\section{Data selection}

Studies were compiled over several years as a result of QMRA research activities related to these and other pathogens, not through a systematic review. Studies were identified through searches of the PubMed and Web of Science databases and by cross-referencing cited references. The databases were queried again prior to drafting this article.

One author (RMJ) reviewed all studies to assess whether studies met the data conditions for dose-response analysis. Data met the following conditions for dose-response analysis: $i$ ) at least three dose levels were used, and $i i$ ) the number doses with response rate other than $0 \%$ or $100 \%$ was equal to or greater than the number of doseresponse model parameters. These conditions are consistent with recommendations by Haas et al. [12]. Qualitatively, data were also evaluated for relevance for human exposures, with consideration for the route of exposure and outcome studied. 


\section{The infectious microbes}

Bordetella pertussis is a bacterium that causes the disease pertussis, commonly known as whooping cough. $B$. pertussis cells adhere to cilia of epithelial cells in the upper respiratory tract, initiating symptoms of infection $[24,25]$. Transmission is generally recognized through the droplet and contact routes [21], but recent animal models suggest that transmission may also occur through the airborne route [26]. As a result, infectivity studies involving aerosol inhalation or deposition into the respiratory tract were judged relevant to disease transmission among humans.

Group A Streptoccocus (GAS) include a number of species, the most common being Streptococcus pyogenes. GAS infections may involve the upper respiratory tract (pharyngitis) or the skin (impetigo), and invasive disease can develop $[27,28]$. Here respiratory infection is considered, associated with droplet transmission [21].

Rhinoviruses are a cause of the common cold. Infection occurs primarily in the upper respiratory tract, though the high temperature in the lower respiratory tract does not fully prevent rhinovirus replication [29]. Studies among human volunteers have found transmission to occur when exposed susceptible volunteers do and do not touch their face $[30,31]$. These data suggest transmission may occur through contact and/or airborne routes, though only droplet transmission is recognized by the CDC [21]. Given these infection sites, experimental exposure to rhinovirus through aerosol and intranasal instillation were judged relevant to natural human infection.

Respiratory syncytial virus (RSV) is a cause of the common cold. In the cotton rat model, virus replication was observed in the alveoli and bronchioles subsequent to intranasal inoculation with RSV [32]. Transmission studies among human volunteers confirmed infection resulting from direct close contact with RSV-infected infants and contact with the eyes and nose after handling RSVcontaminated objects [33]. While the related study of inhalation exposure did not show transmission [33], the result does not preclude transmission through the airborne route due to the potential for low exposure. Virus replication in the alveoli and bronchioles of the cotton rat [32] suggests that inhalation and deposition of RSV in these regions could produce infection. The $\mathrm{CDC}$, however, recognizes only the contract transmission route for RSV [21].

\section{Dose-response models}

Two mechanistic dose-response models were considered: the exponential and beta-Poisson models [12]. The exponential model is a one-hit model, where the probability of infection (or other outcome) given mean dose, $d$, is expressed

$$
P_{I}(d)=1-\exp (-d k)
$$

and $\kappa(0<\kappa \leq 1)$ is the independent and identical probability that an infectious agent survives in the host and initiates infection. The beta-Poisson model is related to the exponential model, but the probability that an infectious agent survives in the host and initiates infection follows a beta distribution. The beta-Poisson model is expressed

$$
P_{I}(d)=1-\left[1+\frac{d}{\beta}\right]^{-\alpha}
$$

where, $\mathrm{d}$ is the mean dose, and $\alpha$ and $\beta$ are parameters of the beta distribution [34]. The median infectious dose, $\mathrm{N}_{50}$, is estimated from $\alpha$ and $\beta$ as:

$$
N_{50}=\frac{\beta}{2^{1 / \alpha}-1}
$$

\section{Dose-response model fit and evaluation}

All analyses were implemented using in-house code written by both authors for the R Project for Statistical Computing. Code is available upon request.

Dose-response models were fitted to data using the method of maximum likelihood. Given an experiment with $i=1,2, \ldots$, D dose levels, $n_{i}$ subjects are given mean dose $d_{\mathrm{i}}$ and $x_{i}$ subjects $\left(0 \leq x_{i} \leq n_{i}\right)$ exhibit the response. The maximum likelihood estimate of the proportion of responding subjects is $\hat{p}_{i}=\frac{x_{i}}{n_{i}}$. The likelihood function is

$$
\operatorname{Lik}(\theta)=\prod_{i=1}^{D}\left(\begin{array}{l}
n_{i} \\
x_{i}
\end{array}\right) p_{i}^{x_{i}}\left(1-p_{i}\right)^{n_{i}-x_{i}}
$$

where $\theta$ is the dose-response model parameter, and $p_{i}$ is the probability of response at dose $d_{i}$ based on the model of choice [12]. For the exponential model, for example, $\theta=\mathrm{K}$ and $p_{i}=1-\exp \left(-d_{i} k\right)$. The most-likely value of $\theta$ maximizes the likelihood function, and minimizes the negative of the log-likelihood function. The latter approach was implemented using the optim function.

Confidence intervals were generated by bootstrapping: Model parameters, $\theta$, were estimated by the method of maximum likelihood to a population randomly sampled with replacement from the study population [12,35]. Given $\mathrm{B}=5,000$ bootstrapped populations, $\theta$ was estimated $\mathrm{B}$ times, $\vec{\theta}=\left\{\hat{\theta}_{1}, \hat{\theta}_{2}, \ldots, \hat{\theta}_{5,000}\right\}$. For the exponential models, the $95 \%$ confidence interval $(\mathrm{CI})$ for the parameter $\mathrm{K}$ is simply the $2.5^{\text {th }}$ and $97.5^{\text {th }}$ percentile of the ordered parameter estimates $\vec{\theta}$. For the beta-Poisson models, the parameters $(\alpha, \beta)$ or $\left(\alpha, \mathrm{N}_{50}\right)$ are jointly distributed [12]. The joint distribution of the $\vec{\theta}$ can be represented graphically. The $95 \% \mathrm{CI}$ in the response was determined by calculating the response at each dose using each of the B values of $\vec{\theta}$, and 
equating the $95 \% \mathrm{CI}$ with the $2.5^{\text {th }}$ and $97.5^{\text {th }}$ percentile of the ordered response values. The $95 \% \mathrm{CI}$ for the response is displayed graphically.

Small sample sizes prevented consistent application of Pearson's $x^{2}$-test to evaluate model fit. Instead, model fit was evaluated with a permutation test with 100,000 permutations of the Boolean responses $[4,36]$. The p-value is the proportion of permutations that yield a log-likelihood larger than that observed with the original data. A large p-value $(p>0.05)$ indicates that the exposure has no effect on the outcome because shuffling the exposures yields log-likelihoods as large or larger as the observed data. Comparison between models was based on Akaike's Information Criterion, AIC $=2 \mathrm{k}-$ $2 \ln (\mathrm{L})$, where $\mathrm{k}$ is the number of parameters in the doseresponse model, and $\ln (\mathrm{L})$ is the $\log$-likelihood $[37,38]$. The AIC penalizes models with more parameters.

\section{Results}

\section{Bordetella pertussis}

Owing to an interest in vaccine development, the infectivity of $B$. pertussis has been evaluated in many animal models, but only three studies in the mouse model met the data selection criteria. The data are presented in Additional file 1: Table S1.

Halperin et al. [39] exposed 10-day old BALB/C mice to $B$. pertussis strain 18323 through aerosol inoculation and intranasal instillation. In the aerosol study, the unit of dose was the concentration of $B$. pertussis cells per $\mathrm{mL}$ of inoculum aerosolized into the exposure chamber. Unfortunately, the concentration of cells in liquid cannot be translated into an airborne concentration or number of inhaled cells, the units of dose relevant to human exposure in the context of disease transmission. As a result, dose-response functions were not fitted to the aerosol study by Halperin et al. [39]. The dose via intranasal instillation was the number of CFU deposited into the nares. Outcomes documented included mortality and positive lung culture upon autopsy: The two outcomes were combined in this analysis to yield a single outcome, infection.

Pittman et al. [40] deposited B. pertussis strain 18323 into the external nares of anesthetized mice (Ham/ICR and Anglia strains) aged four weeks or 6-7 days. The outcome was death, occurring $>2$ hours and $<24$ or 30 days after inoculation. Experiments with mice aged four weeks were pooled because they used the same methods.

Sato et al. [41] exposed mice (DDY and ICR strains) to aerosols of $B$. pertussis Tohama phase I strain during 30 minutes of aerosol generation and 20 minutes of chamber evacuation. Dose was reported as the concentration of $B$. pertussis in the inoculum aerosolized, but in this analysis the dose used was the number of viable cells in the lungs after aerosol exposure, which was determined by autopsy 20 minutes subsequent to exposure. The number of viable cells in the lungs is more directly comparable to human exposure: the fraction of airborne cells that are inhaled and deposit in the lung differ among animals and humans owing to anatomy, respiration rate, animal orientation, etc., but the deposited dose measured in animals can be compared to estimated deposition in the human respiratory tract [42].

For the intranasal studies, AIC recommends the beta-Poisson over the exponential functions (Table 1), which is consistent with graphical display of the models (Additional file 1: Figures S1-S3). In the aerosol study by Sato et al. [41], the two models are functionally equivalent (Additional file 1: Figure S4), and AIC recommends the exponential model because it has only one, rather than two, parameters.

Comparing the two intranasal dose-response studies with mortality outcome, $B$. pertussis appears to be more infectious among younger mice. In the exponential functions, this is indicated by larger values of $\kappa$ (Table 1 ). In the better-fit beta-Poisson functions, infectivity is most readily compared by the $\mathrm{N}_{50}$, which equals $102 \mathrm{CFU}$ and 51,900 CFU for the 6 day old and 4 week old mice, respectively. As expected, the probability of infection is lower than the probability of mortality: For the infection outcome, $\mathrm{N}_{50}=0.632$ CFU. B. pertussis appears substantially less infectious through aerosol exposure, for which $\mathrm{N}_{50}=5.16 \times 10^{6} \mathrm{CFU}$. Differences in infectivity with age have been observed for other organisms, like influenza, in animal models and epidemics [43].

\section{Group A streptococci (GAS)}

Owing to the focus on respiratory infection, human doseresponse studies using intradermal injection [44] and wound inoculation [45] were not evaluated. Exposure though the respiratory tract has only been studied among mice, by Wessels and Bronze [46], who administered GAS (M type 24 strain Vaughn) via intranasal instillation. The study outcomes were mortality and infection, where infection was defined by pharyngeal swab culture positive for GAS (Additional file 1: Table S2). Experiments involving the mutant strain were not used.

For both outcomes, AIC recommends the beta-Poisson model (Table 1). Confidence intervals are narrower for the mortality-based dose-response models than for the infection-based models (Additional file 1: Figures S5 and S6). As indicated by $\mathrm{N}_{50}$, higher doses are required to obtain $50 \%$ mortality than $50 \%$ infectivity (Table 1 ).

\section{Rhinovirus}

Aerosol infectivity studies of human volunteers have little information for dose-response modeling because they observed $100 \%$ of volunteers to become infected: Exposures were $\geq 16 \mathrm{TCID}_{50}$ [47] and $\geq 17 \mathrm{TCID}_{50}$ rhinovirus $\mathrm{NIH}$ 
Table 1 Fitted dose-response models for selected agents

\begin{tabular}{|c|c|c|c|c|c|c|c|c|c|}
\hline \multirow[b]{2}{*}{ Host } & \multirow[b]{2}{*}{ Exposure (unit) } & \multirow[b]{2}{*}{ Outcome } & \multirow[b]{2}{*}{ Ref } & \multicolumn{3}{|l|}{ Exponential Model } & \multicolumn{3}{|c|}{ Beta-Poisson Model } \\
\hline & & & & $\kappa(95 \% \mathrm{Cl})$ & $\mathrm{AIC}^{\mathrm{a}}$ & p-value ${ }^{\mathbf{b}}$ & $\alpha, \beta\left(\mathrm{N}_{50}\right)$ & $\mathrm{AIC}^{\mathrm{a}}$ & p-value \\
\hline \multicolumn{10}{|l|}{ Bordetella pertussis } \\
\hline \multirow[t]{2}{*}{ Mouse (10 day old) } & Intranasal (CFU) & Infection & [39] & $1.14 \times 10^{-8}$ & 607 & 0 & $0.086,2000$ & 50.9 & 0.431 \\
\hline & & & & $\left(6.47 \times 10^{-9}, 3.73 \times 10^{-8}\right)$ & & & $(0.632)$ & & \\
\hline \multirow[t]{2}{*}{ Mouse (4 week old) } & Intranasal (CFU) & Mortality & {$[40]$} & $6.28 \times 10^{-8}$ & 58.9 & 0 & $0.239,8.91 \times 10^{5}$ & 47.3 & 0.972 \\
\hline & & & & $\left(4.85 \times 10^{-8}, 8.75 \times 10^{-8}\right)$ & & & $(51900)$ & & \\
\hline \multirow[t]{2}{*}{ Mouse (6 day old) } & Intranasal (CFU) & Mortality & {$[40]$} & $3.83 \times 10^{-5}$ & 158 & 0 & $0.556,252$ & 24.2 & 0 \\
\hline & & & & $\left(1.52 \times 10^{-5}, 1.04 \times 10^{-3}\right)$ & & & (102) & & \\
\hline \multirow[t]{2}{*}{ Mouse (21 day old) } & Aerosol (CFU inhaled) & Mortality & [41] & $2.15 \times 10^{-6}$ & 8.25 & 0 & $3.34,1.19 \times 10^{6}$ & 10.0 & 0 \\
\hline & & & & $\left(1.10 \times 10^{-6}, 5.76 \times 10^{-6}\right)$ & & & $\left(5.16 \times 10^{6}\right)$ & & \\
\hline \multicolumn{10}{|l|}{ Group A Streptococcus } \\
\hline \multirow[t]{2}{*}{ Mouse } & Intranasal (CFU) & Infection & {$[46]$} & $1.05 \times 10^{-6}$ & 72.8 & 0.088 & $0.341,1260$ & 15.6 & 0.308 \\
\hline & & & & $\left(3.19 \times 10^{-7}, 2.04 \times 10^{-4}\right)$ & & & $(5460)$ & & \\
\hline \multirow[t]{2}{*}{ Mouse } & Intranasal (CFU) & Mortality & {$[46]$} & $1.38 \times 10^{-6}$ & 9.22 & 0 & $0.762,1.98 \times 10^{5}$ & 10.1 & $<0.001$ \\
\hline & & & & $\left(4.59 \times 10^{-7}, 7.44 \times 10^{-6}\right)$ & & & $\left(1.34 \times 10^{5}\right)$ & & \\
\hline \multicolumn{10}{|l|}{ Rhinovirus } \\
\hline \multirow[t]{2}{*}{ Human } & Intranasal $\left(\mathrm{TCID}_{50}\right)$ & Infection & [51] & 1.00 & 11.4 & 0 & $0.725,0.173$ & 12.8 & 0 \\
\hline & & & & $(0.484,1.00)$ & & & $(0.108)$ & & \\
\hline \multirow[t]{2}{*}{ Human (antibody-free) } & Intranasal $\left(\mathrm{TCID}_{50}\right)$ & Infection & {$[50]$} & 0.366 & 75.2 & 0.009 & $0.701,0.111$ & 16.9 & 0.427 \\
\hline & & & & $(0.125,1.00)$ & & & $(0.066)$ & & \\
\hline \multirow{2}{*}{$\begin{array}{l}\text { Human (antibody-free, } \\
\text { pooled) }\end{array}$} & Intranasal $\left(T_{C I D}\right)$ & Infection & {$[50,51]$} & 0.428 & 89.9 & 0 & $0.697,0.121$ & 25.8 & 0 \\
\hline & & & & $(0.154,1.00)$ & & & $(0.071)$ & & \\
\hline \multirow[t]{2}{*}{ Human (antibody $\leq 4$ ) } & Intranasal $\left(\mathrm{TCID}_{50}\right)$ & Infection & {$[50]$} & 0.138 & 123 & 0 & $0.374,0.076$ & 24.0 & 0.358 \\
\hline & & & & $(0.074,0.420)$ & & & $(0.014)$ & & \\
\hline \multicolumn{10}{|l|}{ Respiratory Syncytial Virus } \\
\hline \multirow[t]{2}{*}{ Human } & Intranasal $\left(\mathrm{TCID}_{50}\right)$ & Infection & {$[55]$} & $9.98 \times 10^{-5}$ & 5.92 & 0.002 & $1.14,6610$ & 7.69 & 0.034 \\
\hline & & & & $\left(2.41 \times 10^{-5}, 5.08 \times 10^{-4}\right)$ & & & $(7900)$ & & \\
\hline \multirow[t]{2}{*}{ Human (pooled) } & Intranasal $\left(\mathrm{TCID}_{50}\right)$ & Infection & {$[54,55]$} & $2.34 \times 10^{-5}$ & 20.3 & 0.014 & $0.217,1380$ & 16.0 & 0.080 \\
\hline & & & & $\left(1.18 \times 10^{-5}, 4.42 \times 10^{-5}\right)$ & & & $(59.0)$ & & \\
\hline \multirow[t]{2}{*}{ Human (pooled) } & Intranasal $\left(T C I D_{50}\right)$ & Infection & {$[53-55]$} & $2.86 \times 10^{-5}$ & 95.5 & 0.572 & $0.026,8.59 \times 10^{-8}$ & 39.6 & 0.999 \\
\hline & & & & $\left(1.65 \times 10^{-5}, 5.60 \times 10^{-5}\right)$ & & & $\left(2.27 \times 10^{-19}\right)$ & & \\
\hline
\end{tabular}

${ }^{a}$ AIC is Akaike's Information Criterion.

${ }^{b} p$-value is for the permutation test.

1734 [48]. Two of three intranasal infectivity studies identified met the inclusion criteria and were evaluated (Additional file 1: Table S3) [49-51].

Hendley et al. [50] inoculated volunteers with varied levels of serum antibodies intranasally with rhinovirus type 39 . Higher infectivity was observed among volunteers with lower antibody levels prior to inoculation (Tables 1 and S3), which makes sense because higher antibody levels are indicative of immunity. Regardless of pre-inoculation antibody level (antibody-free or $\leq 4$ ), AIC recommends the beta-Poisson dose-response models (Table 1), which qualitatively provide a better representation of the data than the exponential model (Additional file 1: Figures S7 and S8).
In contrast, AIC recommends the exponential model for intranasal infectivity of rhinovirus type 16 observed by D'Alessio et al. [51]. The maximum likelihood estimate of the exponential model parameter is $\mathrm{K}=1$, indicating that each rhinovirus can initiate infection. As $\mathrm{k}$ must be less than or equal to one, the upper 95\% confidence interval coincides with the maximum likelihood estimate (Additional file 1: Figure S9).

The two studies used different strains of rhinovirus types 16 and 39 , and the values of $\mathrm{K}$ and $\mathrm{N}_{50}$ suggest that type 16 may be more infectious than type 39 (Table 1 ). When data for the two rhinoviruses among antibody-free volunteers were pooled, the fitted dose-response functions 
had $\mathrm{K}$ and $\mathrm{N}_{50}$ values between those observed for each rhinovirus type (Table 1, Additional file 1: Figure S10).

\section{Respiratory syncytial virus}

While numerous animal models have been used to study RSV infection [52], none met the data selection criteria. Of the studies with human volunteers [53-56], only one study met the data selection criteria: Hall et al. [55] inoculated human volunteers with RSV strain A2 in through the nose or eyes (Additional file 1: Table S4). Two other studies $[53,54]$ were considered for pooling with Hall et al. because they used RSV strain A2 and a similar study design (Additional file 1: Table S4).

For the Hall et al. [55] data alone, the two dose-response models are functionally equivalent (Additional file 1: Figure S11), and AIC recommends the exponential model (Table 1). However, when pooled with Lee et al. [54], the beta-Poisson model is recommended by AIC (Table 1, Additional file 1: Figure S12). The addition of observations by Mills et al. [53] offered no improvement, as indicated by the increased p-value and AIC (Table 1, Additional file 1: Figure S13).

\section{Discussion}

In disease transmission, the critical step is that an infectious microbe moves through the environment to a site in a susceptible person where it is capable of initiating infection. To accurately quantify risk of infection, a doseresponse function should be based on experimental data that represent this process. In studies with human volunteers, this means the exposure conditions and doses administered should reflect natural transmission conditions. In studies with animal models, there are additional host-specific concerns. In ideal situations, the infection sites are in similar anatomical locations in the animal model and humans, and the fraction of airborne microbes that reach the infection sites is the same, or can be corrected for based on geometry of the respiratory tract, animal orientation, respiratory rate and other factors. Often, the conditions are not ideal, and important uncertainties are introduced to the extrapolation of an animal-based dose-response function to humans.

Intranasal instillation is an exposure route commonly used in animal models and human subjects, but it is difficult to interpret for natural disease transmission. Intranasal instillation involves the introduction of a small volume of fluid containing infectious microbes into the nares: Given sufficient fluid volume, instilled materials have been shown to move to the lungs $[57,58]$. The disease transmission routes that best align with exposures through intranasal instillation are unclear. In contact transmission, infectious microbes likely deposit on the exterior of the nares and/or on the mucous membranes just inside the nares, surfaces readily accessible to the hands. In droplet transmission, particles containing infectious microbes may project onto or into the nares. In airborne transmission, inhaled particles may deposit throughout the respiratory tract, including the head airways [42]. While the deposition patterns of inhaled particles in the human respiratory tract is well understood [42], the dispersion of the infectious microbes in the head airways subsequent to contact and droplet exposure is unknown. Thus, it is particularly difficult to equate the dose administered through intranasal instillation with exposures through the contact and droplet routes.

Mice were the animal models used in experimental infectivity studies for B. pertussis and GAS (Table 1); and are one of many animal models used to study the infectivity and pathogenesis of infectious microbes, the toxicity of chemicals, and disease. The selection of an animal model considers a variety of factors, including: similarity of the respiratory tract, microbe tropism, immune system and response, and symptom presentation between the animal model and humans (see for example the discussion of guinea pigs and ferrets for influenza [59,60]). The presentation of symptoms in the animal model is very important in studies of disease transmission among animals since the route of transmission should be as similar to that in humans as possible; but is likely less important in evaluating a dose-response function. Unfortunately, unless studies have been performed in both humans and animal models it is not possible to evaluate the equivalency.

The objective of this study was to fit dose-response models for selected microbes capable of infecting the respiratory tract, so as to facilitate QMRA. Multiple functions were identified for B. pertussis, GAS, rhinovirus, and RSV (Table 1). Though statistical criteria, such as AIC, indicate the relative performance of two dose-response functions fitted to a specific data set (exponential or beta-Poisson), the selection of a dose-response function from among those fitted to multiple data sets is a context-specific decision that depends upon the specific question to be addressed through the risk assessment. There are many dimensions in the interpretation of doseresponse functions fitted to data from animal models and human volunteers for natural disease transmission that are more important than questions about the study design (e.g., number of subjects, etc.) and statistical measures of model fit. These dimensions include, but are not limited: tropism of the microbe for the host species, anatomical location of sites susceptible to infection, and the unit of exposure and its relation to the number of infectious microbes that reach sites susceptible to infection.

Owing to the importance of the risk assessment context, the fact that few of the dose-response functions yielded an objectively good representation of the data (see Figures in Additional file 1), differences between strains, and uncertainty about extrapolation of animal- 
based dose-response functions to human disease transmission, we have declined to recommend a single doseresponse function for each of the infectious microbes in this study (Table 1). Future work to understand infection processes in more detail for these microbes is necessary, not only for the sake of general knowledge, but also because the diversity of plausible dose-response functions for each microbe may yield different estimates of infection risk, and different risk management conclusions. Two key data gaps are the fate of microbes deposited onto the facial mucous membranes by contact and droplet spray, and the relative distribution of infection sites in the respiratory tract of animal hosts and humans. Work with influenza virus demonstrates the feasibility of the later studies (see [61]).

Frequently, the beta-Poisson model was preferred over the exponential model (Table 1). This is reassuring because the beta-Poisson model allows for biologically-plausibility inter-individual variability in host susceptibility and virus infectivity. Exponential models were preferred when few subjects and few unique doses were tested: In these conditions, there is insufficient data to characterize inter-individual variability and a model with fewer parameters is preferred by AIC.

Despite the limitations identified for the fitted doseresponse functions (Table 1), the availability of these functions makes it possible to apply QMRA to explore questions of significance to public health decision-making. For example, QMRA can be used in conjunction with exposure models and epidemiologic models to explore the effectiveness of infection control interventions in healthcare settings, including personal protective equipment (PPE) and work practices, like patient isolation and hand hygiene. PPE are specialized clothing or equipment worn by workers or other susceptible people to prevent exposure, including: gloves, gowns, eye protection, respirators, facemasks, etc. Infection control interventions are recommended based on observational studies that indicate association of the intervention(s) with lower risk of infection [21], and while the effectiveness of most interventions are biologically plausible, quantitative measures of effectiveness against specific infectious microbes and contract transmission routes that would help to prioritize interventions are often not available [62]. The limitations of the dose-response functions can be addressed in QMRA through uncertainty analysis, and/or by focusing on the change in risk resulting from interventions rather than the absolute value of risk.

QMRA is an important tool for understanding and mitigating infection risk. While limitations of dose-response functions have been described for these agents, there are also significant limitations and uncertainties about other aspects of QMRA, including exposure assessment. Quantitative measurements of infectious microbe emission and exposure are extremely limited, so the parameterization and verification of exposure models are difficult. Nonetheless, QMRA provides a transparent, systematic process by which to understand and evaluate risks posed by infectious microbes. With the new dose-response functions presented herein, the application of QMRA to pertussis, GAS pharyngitis, and viruses causing the common cold can begin.

\section{Conclusions}

Dose-response functions were fitted for B. pertussis, GAS, rhinovirus, and RSV; though all functions have limitations associated with the use of an animal model, intranasal instillation, susceptibility of the hosts, etc. The persistence of these microbes as threats to public health, however, motivate application of QMRA to understand the magnitude of infection risks and identify effective controls to reduce risk. Using the dose-response functions fitted in this study, QMRA can begin to be applied.

\section{Additional file}

Additional file 1: Supplementary materials. The supplementary materials contains the dose-response data analyzed and graphical prescutations of model fit.

\section{Abbreviations}

AIC: Akaike's Information Criterion; CDC: U.S. Centers for Disease Control and Prevention; GAS: Group A Streptococcus; PPE: Personal Protective Equipment; QMRA: Quantitative microbial risk assessment; RSV: Respiratory Syncytial Virus.

\section{Competing interests}

The authors declare that they have no competing interests.

\section{Authors' contributions}

RMJ planned the study, performed statistical analysis and drafted the manuscript. YMS performed statistical analysis and helped to draft the manuscript. Both authors read and approved the final manuscript.

\section{Authors' information}

RMJ is an Assistant Professor in Occupational and Environmental Health Sciences, where she teaches and conducts research in the assessment of human exposures to environmental contaminants, including infectious agents, and risk of adverse health outcomes, such as infection.

\section{Acknowledgements}

This work was supported in part by Eastern Research Group, Inc agreements 0306.00.008/01 and 0306.01.014/01 and National Institute for Occupational Safety and Health Contract 211-2006-M-162553. The funders did not participate in the design of this study; in the collection, analysis and interpretation of the data; in the writing of the manuscript; nor in the decision to submit the manuscript for publication. Opinions expressed in this work are those of the authors. We also acknowledge the Research Open Acess Publishing (ROAPP) Fund of the University of Illinois at Chicago for financial support towards the open access publishing fee for this article.

Received: 22 May 2014 Accepted: 12 February 2015

Published online: 24 February 2015

\section{References}

1. Ashbolt NJ, Schoen ME, Soller JA, Roser DJ. Predicting pathogen risks to aid beach management: The real value of quantitative microbial risk assessment (QMRA). Water Res. 2010;44:4692-703. 
2. Latorre AA, Pradhan AK, Van Kessel JAS, Karns JS, Boor KJ, Rice DH, et al. Quantiative risk assessment of literiosis due to consumption of raw milk. J Food Protection. 2011;74:1268-81.

3. Guillier L, Danan C, Bergis H, Delignette-Muller ML, Granier S, Rudelle S, et al. Use of quantitative microbial risk assessment when investigating foodborne illness outbreaks: The example of a monophasic Salmoella typhimurium 4,5,12:i: - outbreak implicating beef burgers. Intl J Food Microbiol. 2013;166:471-8

4. Nicas $M$, Jones RM. Relative contributions of four exposure pathways to influenza infection risk. Risk Anal. 2009;29:1292-303.

5. Gupta JK, Lin CH, Chen Q. Risk assessment of airborne infectious diseases in aircraft cabins. Indoor Air. 2012;22:388-95.

6. Knibbs LD, Morawska L, Bell SC. The risk of airborne influenza transmission in passenger cars. Epidemiol Infect. 2012;140:474-8.

7. Zhao J, Eisenberg JE, Spicknall IH, Li S, Koopman JS. Model analysis of fomite mediated influenza transmission. PLoS One. 2012;7:e51984.

8. Atkinson MP, Wein LM. Quantifying the routes of transmission for pandemic influenza. Bull Math Biol. 2008;70(3):820-67.

9. Ko G, Thompson KM, Nardell EA. Estimation of tuberculosis risk on a commercial airliner. Risk Anal. 2004;24(2):379-88.

10. Jones RM, Masago Y, Bartrand T, Haas CN, Nicas M, Rose JB. Characterizing the risk of infection from Mycobacterium tuberculosis in commercial passenger aircraft using quantitative microbial risk assessment. Risk Anal. 2009;29(3):355-65.

11. Chen SC, Liao CM, Li SS, You SH. A probabilistic transmission model to assess infection risk from Mycobacterium tuberculosis in commercial passenger trains. Risk Anal. 2011;31:930-9.

12. Haas CN, Rose JB, Gerba CP. Quantitative Microbial Risk Assessment. New York: John Wiley \& Sons; 1999.

13. Beggs CB, Shepherd SJ, Kerr KG. Potential for airborne transmission of infection in the waiting areas of healthcare premises: stochastic analysis using a Monte Carlo model. BMC Infect Dis. 2010;10:247.

14. Sze-To GN, Chao CYH. Use of risk assessment and likelihood estimation to analyze spatial distribution pattern of respiratory infection cases. Risk Anal. 2011;31:351-69.

15. Knibbs LD, Morawska L, Bell SC, Grzybowski P. Room ventilation and the risk of airborne infection transmission in three health care settings within a large teaching hospital. Am J Infect Control. 2011;39:866-72.

16. Wells WF. Airborne contagion and air hygiene: an ecological study of droplet infections. Boston, MA: Harvard University Press; 1955

17. Escombe AR, Oeser C, Gilman RH, Navincopa M, Ticona E, Martinez C, et al. The detection of airborne transmission of tuberculosis from HIV-infected patients, using an in vivo air sampling model. Clin Infect Dis. 2007;44(10):1349-57.

18. Riley EC, Murphy G, Riley RL. Airborne spread of measles in a suburban elementary school. Am J Epidemiol. 1978;107:421-32.

19. Sze-To GN, Chao CYH. Review and comparison between the Wells-Riley and dose-response approaches to risk assessment of infectious respiratory diseases. Indoor Air. 2010;20:2-16.

20. Rudnick SN, Milton DK. Risk of indoor airborne infection transmission estimated from carbon dioxide concentration. Indoor Air. 2003;13:237-45.

21. Siegel JD, Rhinehart $E$, Jackson M, Chiarello $L$, and the Healthcare Infection Control Practices Advisory Committee: 2007 Guidelines for Isolation Precautions: Preventing Transmission of Infectious Agents in Healthcare Settings. http://www.cdc.gov/hicpac/pdf/isolation/isolation2007.pdf.

22. [http://www.cidrap.umn.edu/news-perspective/2014/09/commentary-healthworkers-need-optimal-respiratory-protection-ebola]

23. Roy CJ, Milton DK. Airborne transmission of communicable infection - the elusive pathway. N Engl J Med. 2004;17:1710-2.

24. Mattoo S, Cherry JD. Molecular pathogenesis, epidemiology and clinical manifestations of respiratory infections due to Bordetella pertussis and other Bordetella subspecies. Clin Microbiol Rev. 2005;18:326-82.

25. Lamberti Y, Gorgojo J, Massillo C, Rodriguez ME. Bordetella pertussis entry into respiratory epithelial cells and intracellular survival. Pathog Dis. 2013;69:194-204.

26. Warfel JM, Beren J, Merkel TJ. Airborne transmission of Bordetlla pertussis. J Infect Dis. 2012;206:902-6.

27. [http://www.cdc.gov/groupAstrep/about/faqs.html]

28. Wannamker LW. Differences between streptococcal infections of the throat and of the skin (second of two parts). N Engl J Med. 1970;282:78-85.

29. Kennedy JL, Turner RB, Braciale T, Heymann PW, Borish L. Pathogenesis of rhinovirus infection. Curr Opin Virol. 2012;2:287-93.
30. Dick EC, Jennings LC, Mink KA, Wartgow CD, Inhorn SL. Aerosol transmission of rhinovirus colds. J Infect Dis. 1987;156(3):442-8.

31. Gwaltney Jr JM, Hendley JO. Transmission of experimental rhinovirus infection by contaminated surfaces. Am J Epidemiol. 1982;116:828-33.

32. Murphy BR, Prince GA, Lawrence LA, Croen KD, Collins PL. Detection of respiratory syncytial virus (RSV) infected cells by in situ hybridization in the lungs of cotton rats immunized with formalin-inactivated virus or purified RSV F and G glycoprotein subunit vaccine and challenged with RSV. Virus Res. 1990;16:153-62.

33. Hall CB, Douglas Jr RG. Modes of transmission of respiratory syncytial virus. J Pediatr. 1981;99:100-2.

34. Furumoto WA, Mickey R. A mathematical model for the infectivity-dilution curve of tobacco mosaic virus: experimental tests. Virology. 1967;32(2):224-33.

35. Jones RM, Nicas M, Hubbard AE, Reingold AL. The infectious dose of Coxiella burnetii (Q fever). Appl Biosaf. 2006;11:32-41.

36. Efron B, Tibshirani RJ. An introduction to the bootstrap. Chapman \& Hall/CRC: Boca Raton, FL; 1994.

37. Akaike H. Likelihood of a model and information criteria. J Econom. 1981;16:3-14.

38. Burnham KP, Anderson DR, Huyvaert KP. AIC model selection and multimodel inference in behavioral ecology: some background, observations, and comparisons. Behav Ecol Sociogiol. 2011;65:23-35.

39. Halperin SA, Heifetz SA, Kasina A. Experimental respiratory infection with Bordetella pertussis in mice: comparison of two methods. Clin Invest Med. 1988;11:297-303.

40. Pittman M, Furman BL, Wardlaw AC. Bordetella pertussis respiratory tract infection in the mouse: pathophysiological responses. J Infect Dis. 1980;142:56-66.

41. Sato $Y$, Izumiya K, Sato H, Cowell $J$, Manclark CR. Aerosol infection of mice with Bordetella pertussis. Infect Immun. 1980;29:261-6.

42. Hinds WC. Aerosol Technology. 2nd ed. New York, NY: John Wiley \& Sons; 1999

43. Sun S, Zhao G, Xiao W, Hu J, Guo Y, Yu H, et al. Age-related sensitivity and pathological differences in infections by 2009 pandemic influenza A (H1N1) virus. Virol J. 2011:8(1):52

44. Elek SD, Conen PE. The virulence of Staphylococcus pyogenes for man: A study of the problems of wound infection. Br J Exp Pathol. 1957;38:573-86.

45. Leyden JJ, Stewart R, Kligman AM. Experimental infections with group A streptococci in humans. J Invest Dermatol. 1980;75:196-201.

46. Wessels MR, Bronze MS. Critical role of the group A streptococcal capsule in pharyngeal colonization and infection in mice. Proc Natl Acad Sci U S A. 1994;91:12239-42

47. Cate TR, Couch RB, Fleet WF, Griffith WR, Gerone PJ, Knight V. Production of tracehobronchitis in volunteers with rhinovirus in a small-particle aerosol. Am J Epidemiol. 1965;81:95-105.

48. Douglas Jr RG, Cate TR, Gerone PJ, Couch RB. Quantitative rhinovirus shedding patterns in volunteers. Am Rev Resp Dis. 1966;94:159-67.

49. Cate TR, Couch RB, Johnson KM. Studies with rhinoviruses in volunteers: production of illness, effect of naturally acquired antibody, and demonstration of a protective effect not associated with serum antibody. J Clin Invest. 1964;43:56-67.

50. Hendley JO, Edmondson Jr WP, Gwaltney Jr JM. Relation between naturally acquired immunity and infectivity of two rhinoviruses in volunteers. J Infect Dis. 1972;125:243-8.

51. D'Alessio DJ, Meschievitz CK, Peterson JA, Dick CR, Dick EC. Short-duration exposure and the transmission of rhinoviral colds. J Infect Dis. 1984;150(2):189-94.

52. Byrd LG, Prince GA. Animal models of respiratory syncytial virus infection. Clin Infect Dis. 1997;25:1363-8.

53. Mills J, Van Kirk JE, Wright PF, Chanock RM. Experimental respiratory syncytial virus infection of adults. J Imunol. 1971;107:123-30.

54. Lee EH, Walsh EE, Falsey AR, Betts RF, Treanor JJ. Experimental infection of humans with A2 respiratory syncytial virus. Antiviral Res. 2004;63:191-6.

55. Hall CB, Douglas Jr RG, Schnabel KC, Geiman JM. Infectivity of respiratory syncytial virus by various routes of inoculation. Infect Immun. 1981;33:779-83.

56. Buchman CA, Doyle WJ, Pilcher O, Gentile DA, Skoner DP. Nasal and otologic effects of experimental respiratory syncytial virus infection in adults. Am J Otolaryngol. 2002;23:70-5.

57. Southam DS, Dolovich M, O'Byrne PM, Inman MD. Distribution of intranasal instillations in mice: effects of volume, time, body position and anesthesia. Am J Phiol Loung cell Mol Physiol. 2002;282:L833-9.

58. Miller MA, Stabenow JM, Parvathareddy J, Wodowski AJ, Fabrizio TP, Bina XR, et al. Visualization of murine intranasal dosing efficiency using luminescent Francisella tularensis: effect of instillation volume and form of anesthesia. PLoS One. 2012;7:e31359 
59. Belser JA, Katz JM, Tumpey TM. The ferret as a model organism to study influenza A virus infection. Dis Model Mech. 2011;4:575-9.

60. Lowen AC, Mubareka S, Tumpey TM, Garcia-Sastre A, Palese P. The guinea pig as a transmission model for human influenza viruses. Proc Natl Acad Sci U S A. 2006;103(26):9988-92.

61. Lakdawala SS, Shih AR, Jayaraman A, Lamirande EW, Moore I, Paskel M, et al. Receptor specificity does not affect replication or virulence of the 2009 pandemic H1N1 influenza virus in mice and ferrets. Virology. 2013:446:349-56.

62. Larson EL, Liverman CT. Preventing Transmission of Pandemic Influenza and Other Viral Respiratory Diseases: Personal Protective Equipment for Healthcare Workers: Update 2010. Washington, D.C: National Academy of Sciences; 2011.

\section{Submit your next manuscript to BioMed Central and take full advantage of:}

- Convenient online submission

- Thorough peer review

- No space constraints or color figure charges

- Immediate publication on acceptance

- Inclusion in PubMed, CAS, Scopus and Google Scholar

- Research which is freely available for redistribution 